\title{
Upcoming Meetings
}

\section{AOrTA}

AORTA, August 2015, Volume 3, Issue 4: 151

\section{List of Upcoming Meetings}

\section{January 2016}

1. Controversies and Updates in Vascular Surgery January 21-23, 2016

Paris, France

Meeting information available at:

www.cacvs.org

2. Society of Thoracic Surgeons 52nd Annual Meeting and STS/AATS Tech-Con 2016

January 23-27, 2016

Phoenix, Arizona

Meeting information available at:

www.sts.org/annualmeeting

\section{February 2016}

1. Aortic Valve Repair: A Step by Step Approach L'Institut Mutualiste Montsouris

February 4-5, 2016

Paris, France

Meeting information available at:

www.caviaar.com

2. 62 nd Annual Meeting of Indian Association of Cardiovascular and Thoracic Surgeons

February 18-21, 2016

Lucknow, UP, India

Meeting information available at:

www.iactsconlucknow2016.org

3. University of Virginia 8th Annual Cardiac Symposium: Debates and Controversies in Cardiovascular Disease

February 25-27, 2016

Wintergreen, VA

Meeting information available at:

www.cmevillage.com
4. 34th Cardiovascular Surgical Symposium (CSS) February 27-March 5, 2016

Zurs, Austria

Meeting information available at:

www.surgery-zurs.at

\section{March 2016}

1. 12th International Congress of Update in Cardiology and Cardiovascular Surgery March 10-13, 2016

Antalya, Turkey

Meeting information available at:

www.uccvs2016.org

2. Society for Cardiothoracic Surgery in Great Britain \& Ireland 80th Annual Meeting

March 13-15, 2016

Birmingham, United Kingdom

Meeting information available at:

www.scts.org 